\title{
Observational aspects of Outbursting Black Hole Sources - Evolution of Spectro-Temporal features and X-ray Variability
}

\author{
Sreehari H. ${ }^{1,2, *}$, Anuj Nandi ${ }^{1}$, Radhika D. ${ }^{3}$, Nirmal Iyer ${ }^{4} \&$ Samir Mandal $^{5}$ \\ ${ }^{1}$ Space Astronomy Group, ISITE campus, ISRO Satellite Centre, Outer Ring Road, Marathahalli, Bengaluru, \\ 560037, India. \\ ${ }^{2}$ Department of Physics, Indian Institute of Science, Bengaluru, 560012, India. \\ ${ }^{3}$ Department of Physics, Dayananda Sagar University, Bengaluru, 560068, India. \\ ${ }^{4}$ Albanova university centre, KTH PAP, Stockholm 10691, Sweden. \\ ${ }^{5}$ Department of Physics, Indian Institute of Space Science and Technology, Trivandrum, 695547, India.
}

"Corresponding author. E-mail: hjsreehari@gmail.com

MS received 28 August 2017; revised 28 August 2017; accepted 28 August 2017

\begin{abstract}
We report on our attempt to understand the outbursting profile of Galactic Black Hole (GBH) sources, keeping in mind the evolution of temporal and spectral features during the outburst. We present results of evolution of Quasi-periodic Oscillations (QPOs), spectral states and possible connection with Jet ejections during the outburst phase. Further, we attempt to connect the observed X-ray variabilities (i.e., 'class' / 'structured' variabilities, similar to GRS 1915+105) with spectral states of BH sources. Towards these studies, we consider three Black Hole sources that have undergone single (XTE J1859+226), a few (IGR J17091-3624) and many (GX 339-4) outbursts since the start of RXTE era. Finally, we model the broadband energy spectra $(3-150 \mathrm{keV})$ of different spectral states using RXTE and NUSTAR observations. Results are discussed in the context of two component advective flow model, while constraining the mass of the three BH sources.
\end{abstract}

Key words. accretion—X-ray binaries—black holes—ISM:jets and outflows—radiation mechanisms

\section{Introduction}

A Galactic Black Hole (GBH) binary system consists of a primary black hole and a secondary star. If the secondary star is a Sun like star with mass of a few solar masses, it is called a Low Mass X-ray Binary (LMXB) and if the companion star is a few tens of solar masses in size, it is a High Mass X-ray Binary (HMXB). The primary accretes matter from the secondary star via Roche lobe over flow in the case of LMXBs and from stellar winds in the case of HMXBs. A few X-ray binaries also have intermediate mass companions and such systems are termed Intermediate Mass X-Ray binaries (IMXBs) (Podsiadlowski et al. 2002).

Tetarenko et al. (2016) has reported 77 GBH sources while Corral-Santana et al. (2015) reported 59 transient GBH sources of which 18 are dynamically confirmed. Till date, three extragalactic $\mathrm{BH}$ transients also have been observed. They are LMC X-1, LMC X-3 and M33 X-7 (see Corral-Santana et al. (2015) for details). Besides these, there are Ultra-luminous X-ray sources (ULXs) which may harbour intermediate mass black holes (Feng \& Soria 2011). GBH systems can be either persistent or outbursting (Tanaka \& Shibazaki 1996). Some GBHs like Cyg X-1 shows persistent Xray emission while some others like GRS $1915+105$ are persistent with aperiodic X-ray variability. Sources like GX 339-4 undergo frequent outbursts separated by quiescent phases. There are also sources like GRO J165540 that remain mostly in the quiescent phase and goes into outburst once in a decade or so.

During an outburst a typical black hole binary system goes through different canonical states (Homan \& Belloni 2005; Belloni et al. 2005; Remillard \& McClintock 2006; Nandi et al. 2012). In general, states are classified as Low-Hard State (LHS), Hard-Intermediate State (HIMS), Soft-Intermediate State (SIMS) and the High-Soft State (HSS). It is the mass transfer rate onto the $\mathrm{BH}$ which determines the transient behaviour of the source (Tanaka \& Lewin 1995). During the LHS, the source energy spectrum can be modelled mainly with a powerlaw component along with a gaussian (for the iron line) and reflection components. The presence of a disk is usually seen as the system undergoes transition into the intermediate states and the disk contribution increases significantly as the source enters the 
HSS. Meanwhile, the powerlaw index also increases from around 1.5 in the LHS to values as high as 2.8 in the HSS.

One can study the state evolution of BH binaries associated with the various branches of the 'q-diagram' or Hardness Intensity Diagram (HID) observed during outburst. The HID of a typical outburst is a hysteresis loop in the shape of a 'q' (Homan \& Belloni 2005). From the HID, it is evident that the decay phase of the outburst always has lower total flux values as compared to the rising phase.

As the system evolves through different states, variation in its temporal characteristics has been observed. Specifically, we see different types of Quasi-periodic Oscillations (QPOs) in the power spectrum of the source. Low Frequency QPOs (LFQPOs) are generally found in the LHS, HIMS and SIMS. They are categorised into Type A, Type B and Type C based on the frequency of oscillation, rms value, quality factor, and significance. Casella et al. (2004) has given a detailed classification of LFQPOs. A few black hole binary systems also exhibit High Frequency QPOs (HFQPOs). But in this paper, we restrict our study only to C-Type LFQPOs as they show evolution in their frequencies during the rising and decay phases of an outburst.

$\mathrm{BH}$ binaries (BHBs) also exhibit different types of $\mathrm{X}$-ray variability in their light curves. Some sources like GRS 1915+105 are known to exhibit 'structured' or 'class' variability. Apart from X-ray outbursts and variabilities in smaller time scales, BHBs are also observed to exhibit bipolar radio jet emissions (Fender $e t$ al. 2004).

\subsection{Motivation and Source Selection}

The motivation of this work is to do a comparative study of the outburst profiles, QPO evolution and evolution of X-ray spectral features of three BH binary sources namely XTE J1859+226, GX 339-4 and IGR J170913624. These three sources have also shown signature of X-ray variability during their outburst phases. We compare these variability with the structured variability exhibited by the source GRS $1915+105$.

XTE J1859+226 has gone into an outburst only in 1999 following which it has been in quiescence. GX 339-4 is one of the most active transient sources which has undergone several outbursts in the RXTE-SWIFT era. Meanwhile, IGR J17091-3624 undergoes an outburst approximately once in every four years since its discovery in 2003. Hence, we have chosen these three BHBs which differ in their frequency of outbursts. Figure 1 1 shows the frequency of outbursts of three different BH sources.

Besides this all three sources have shown evolution of Low Frequency QPOs and we could model them with the Propagating Oscillatory Shock (POS) model. Also, we have done spectral modelling of the four different states of the BH binary GX 339-4 using two component advective flow model. For this, we have chosen only the 2002 outburst of GX 339-4 as a representative case. The modelling with phenomenological models as well as with the two component flow for the hard and soft states of the other two sources (XTE J1859+226 and IGR J17091-3624) are also shown in this paper. Estimation of mass of the black holes for these sources are also done using the two component flow model. Detailed modelling of the entire outbursts of these two sources is going on and will be published elsewhere. We have also compared the variability exhibited by the three sources we consider with that exhibited by GRS $1915+105$, which is known for the structured variability that it exhibits.

We also intend to study the possible connection between X-ray variability and state classification of the sources there by interpreting the state of GRS 1915+105. This is done based on the states of the three BHBs (XTE J1859+226, GX 339-4 and IGR J17091-3624) during the period in which they exhibit variability.

\section{Observation, Analysis and Modelling}

We have considered the observations of different $\mathrm{BH}$ binaries carried out with the All Sky Monitor (ASM), Proportional Counter Array (PCA) and the High Energy X-ray Timing Experiment (HEXTE) aboard the satellite Rossi X-ray Timing Explorer (RXTE). The ASM operated in the energy range of $1.3 \mathrm{keV}$ to $12.2 \mathrm{keV}$ and we obtain the outburst profile of various sources using data from this instrument. PCA data in the energy range of 2 to $60 \mathrm{keV}$ and HEXTE data in 15 to $200 \mathrm{keV}$ were usually considered for spectral studies. We extract the energy spectrum from PCA and HEXTE using standard techniques. The PCA data is used for both spectral and temporal analysis while HEXTE is used mainly for generating energy spectrum at higher energies. Broadband energy spectrum combining PCA and HEXTE is modelled to study the state evolution of different BH binary systems.

Besides the spectral studies, we also search PCA data for the presence of peaked noise components or QPOs. The science event files are used to extract the light curves which are used to generate the power spectrum. We model the obtained power spectra with powerlaws and/or Lorentzians. If any QPO like feature is found then it is fit with Lorentzians. The significance of QPOs in the spectrum are calculated as the ratio of norm to its negative error. If significance $>3$ and the Quality factor, $Q=v / F W H M$ is found to be greater 

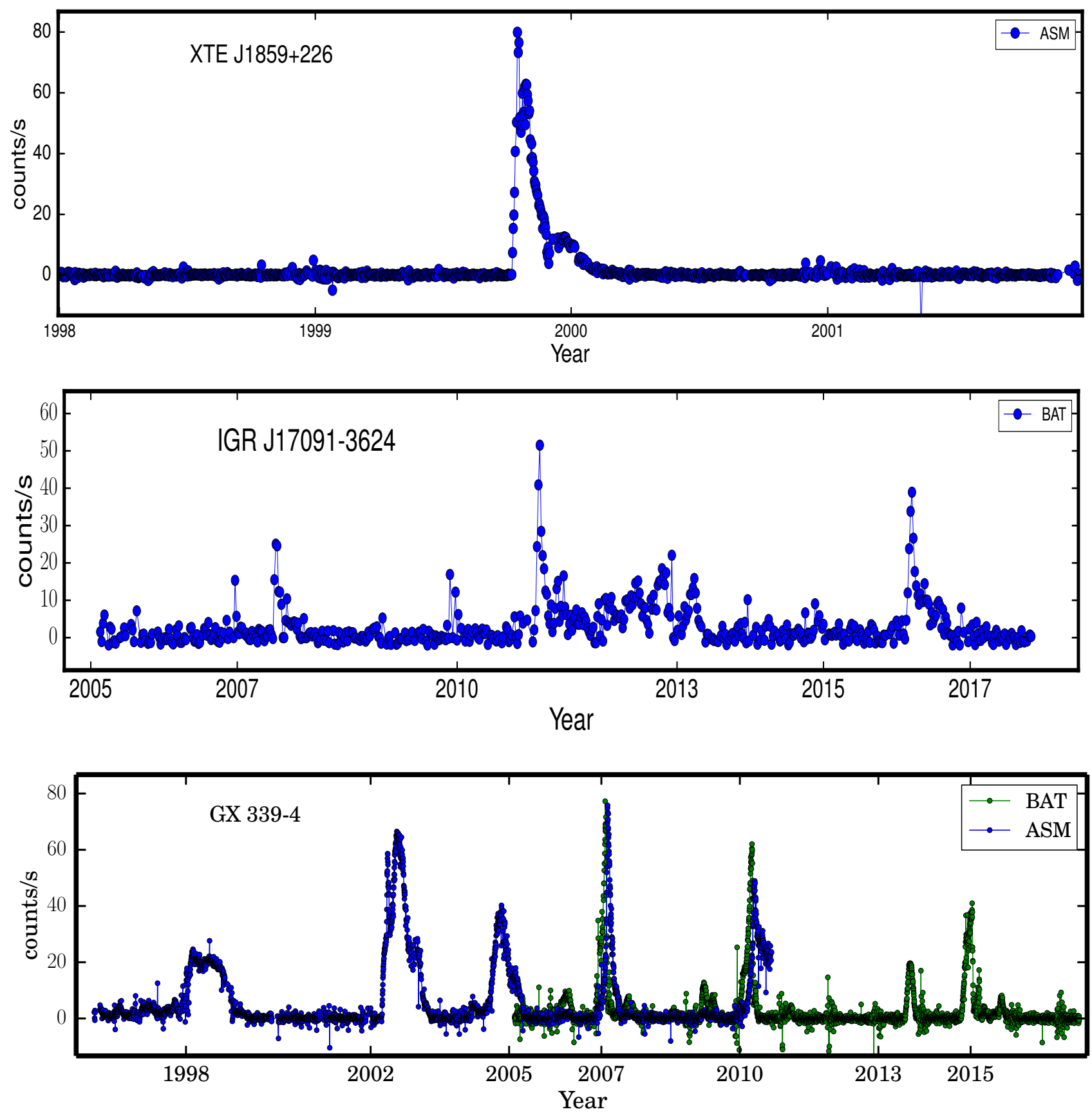

Figure 1. Light curve of some outbursting GBHs showing that the frequency of outburst varies from source to source. XTE $\mathrm{J} 1859+226$ has had only one outburst in the last two decades. GX 339-4 goes into outburst once in every two or three years while IGR J17091-3624 clearly shows outbursts in 2007, 2011 and 2016. 
than 2 then the narrow feature is considered as a QPO. QPOs are classified into low frequency QPOs (LFQPOs) and high frequency QPOs (HFQPOs) based on their frequency ranges. Typically LFQPOs appear in the frequency range of 0.1 to $30 \mathrm{~Hz}$ and HFQPOs appear at frequencies more than $30 \mathrm{~Hz}$ and extends upto $\approx$ $500 \mathrm{~Hz}$ in the case of BH binaries. The highest HFQPO observed so far in $\mathrm{BH}$ binaries is from the source GRO $\mathrm{J} 1655-40$ and it is $\sim 450 \mathrm{~Hz}$ (Strohmayer 2001).

We also analysed SWIFT - XRT data of IGR J170913624 and studied the variability of the source. XRT operates in the range from 0.5 to $10 \mathrm{keV}$. xrtpipeline was run to obtain cleaned event files which were then filtered corresponding to grades 0-2 using XSELECT software. We chose a circular region of 30 arc seconds centred at the source RA and DEC to obtain the source region and an annular region of 60 arc second inner radius and 90 arc second outer radius as the background region (see Radhika et al. 2016b for further details). Then we extract light curves and energy spectra corresponding to these source and background regions. The background subtracted light curves are then used to produce the power spectra for further analysis. We use xrtmkarf to generate the arf files and then we rebin the extracted source energy spectra to 25 counts per bin with the ftool grppha.

Six TOO observations with NuSTAR are also available for the 2016 outburst of IGR J17091-3624. We include spectral studies based on two of those (from LHS and SIMS) in this paper. The other four broadband spectra are studied in detail by Radhika et al. (2018). We use nupipeline to extract the level 2 data based on the procedure given in NuSTAR guide 1. A circular region of $30^{\prime \prime}$ centred at the source RA and DEC is used as the source region and another 30" circle far away from the source is taken as the background region. Then we use the ftool nuproducts to extract the spectrum, response and arf files. The obtained spectrum is re-binned to 30 counts per bin. In the next section, we present the results of the data analysis and modelling done with the three sources under consideration.

\section{Results: Spectro-Temporal features of Outburst- ing BH Sources}

In order to study the spectro-temporal features of outbursting BH sources, we consider three BH sources, namely XTE J1859+226, GX 339-4 and IGR J170913624. The X-ray transient source XTE J1859+226 was discovered with ASM onboard RXTE on Oct 9, 1999

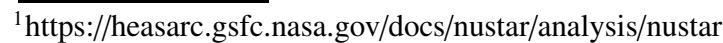
swguide.pdf
}

(Wood et al. 1999). Detailed studies of the outburst had revealed the spectral state characteristics of the source (Homan \& Belloni 2005; Radhika \& Nandi 2014). GX $339-4$ is a well known transient source and has undergone several outbursts during the RXTE -SWIFT era (See Figure 1). Detailed study of its spectral and temporal evolution have paved way for understanding the general characteristics of GBH transients (Motta et al. 2011; Nandi et al. 2012). The source IGR J17091-3624 was discovered by the International Gamma-ray Astrophysics Laboratory (INTEGRAL) in the year 2003 and also exhibited multiple outbursts. A very detailed study of the source has revealed its spectral and temporal properties (Capitanio et al. 2006; Capitanio et al. 2012; Iyer et al. 2015). The most significant feature of the source is the variabilities/oscillations discovered in its light curve during the 2011 outburst (Altamirano et al. 2011). These have been found to be similar to the well known source GRS 1915+105 (Belloni et al. 2001). In the following subsections, we present results and discuss the Outburst profile, evolution of QPO frequencies, Spectral state evolution, Jet connection with spectral states, $\mathrm{X}$-ray variability and broadband spectral modelling based on two component flow model of $\mathrm{BH}$ binary sources.

\subsection{Outburst Profile}

Outbursting BH sources either have a Fast Rise Exponential Decay (FRED) or a Slow Rise Exponential Decay (SRED) profile. In Figure 2, we present a typical FRED outburst profile and the corresponding qdiagram exhibited by the microquasar XTE J1859+226 in the year 1999. The q-diagram or the HID is the plot of total flux versus hardness ratio (ratio of flux in higher energy range to that in lower energy range). $\mathrm{BH}$ binaries (BHB) undergo state transitions from hard to soft state as the outburst progresses. Hard states correspond to observations with considerable amount of high energy photons while the soft states are mostly dominated by thermal emission with less contribution from high energy photons. A typical outbursting $\mathrm{BH}$ binary will go through different spectral states (see for details Homan et al. 2001; Belloni et al. 2005; Remillard \& McClintock, 2006; Nandi et al. 2012) during the outburst phase. The outburst profile and Q diagram for XTE J1859+226 is shown in Figure 2, We chose the outburst of XTE J1859+226 as it is a 'good' representative for the outburst and q-profiles for BHBs. It evolves through all the canonical states and hence we have included only this profile in the paper. Similar plots for GX 339-4 and IGR J17091-3624 will be presented elsewhere.

As the outburst begins, the source is in LHS where the hardness ratio is maximum. This is followed by the 

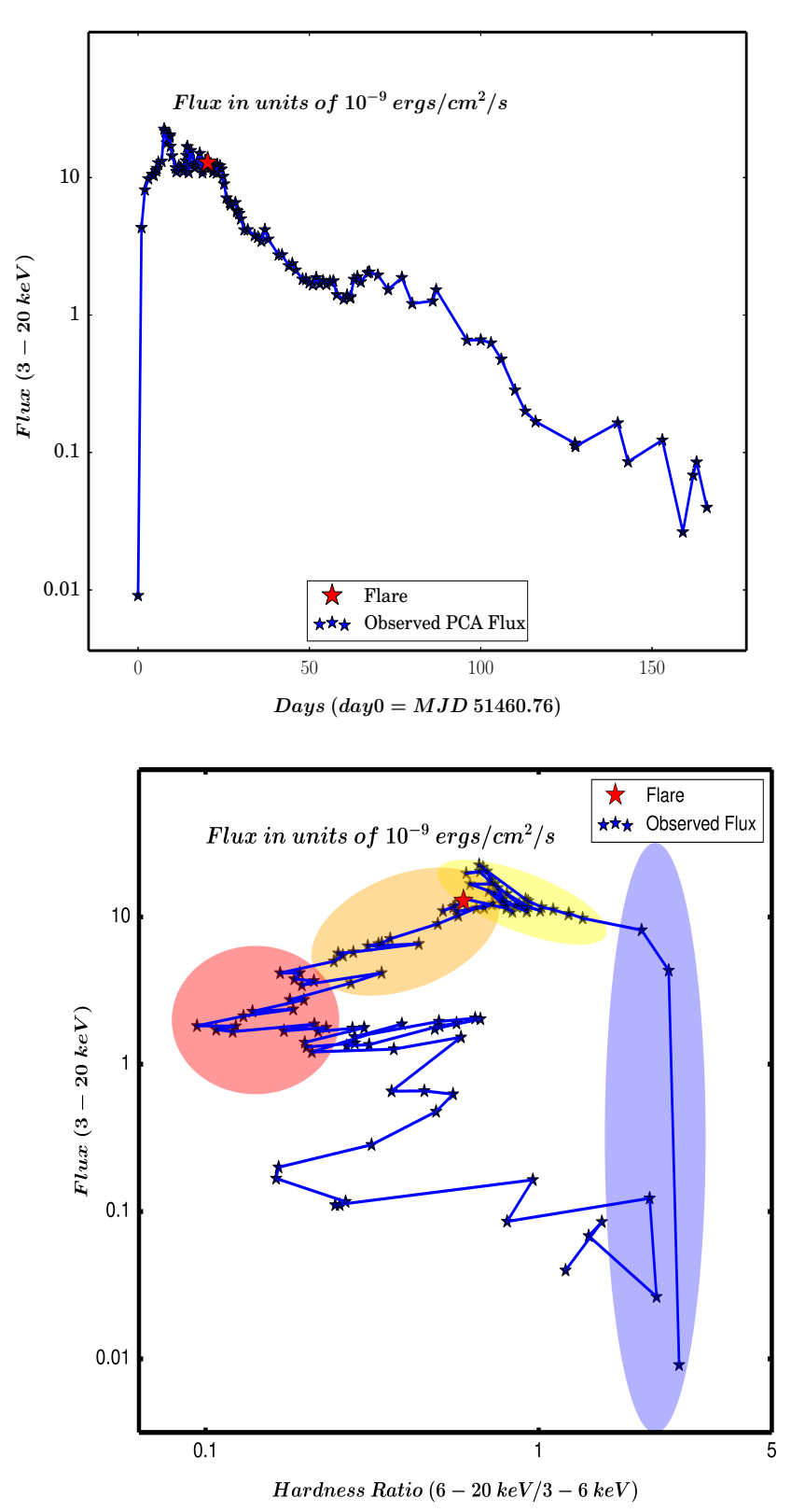

Figure 2. The top panel shows the outburst profile of the source XTE J1859+226 during its 1999 outburst. It is a typical FRED profile with the rising phase extending only around 30 days while the decay phase is gradual and takes more than 120 days. The red marker corresponds to a radio flare observed during this outburst. The corresponding q-plot is shown in the bottom panel. The blue stars indicate the observed flux versus Hardness ratio. The rising phase states are marked in coloured patches. The LHS in blue patch, HIMS in yellow patch, SIMS in orange patch and HSS in red patch. We also show the occurrence of a radio flare in both outburst profile and in HID, marked with a red color star.
HIMS where the photon counts are high and there is significant contribution from the higher energies. The source then enters the SIMS where the hardness ratio reduces and the soft flux increases. When the source is in the HSS, the hardness ratio reaches its smallest value and the spectrum is dominated by thermal emission. In the declining phase, the source again occupies the SIMS, HIMS and LHS with similar values for the hardness ratio as was the case for the rising phase. In Figure 2, we show the rising phase LHS in blue patch, HIMS in yellow patch, SIMS in orange patch and HSS in red patch. After the HSS, the source enters the decay phase. The decay phase characteristics are similar to that of the corresponding states in the rising phase except for a decrease in total flux values. We also note that during the LHS and HIMS of both rising and decay phases, LFQPOs are detected. They generally evolve from around $0.1 \mathrm{~Hz}$ to a maximum of around 20 to 30 $\mathrm{Hz}$. Here, we study the evolution of LFQPO in the rising phase.

\subsection{Evolution of LFQPOs}

Outbursting BH sources exhibit QPOs which are features that peak in the power spectrum generated from source light curves. Typically LFQPOs are of three types C, B and A (Casella et al. 2004). C type LFQPOs appear in the LHS and HIMS only. A and B types are of lesser rms values and are seen in the SIMS. Ctype QPOs appear in power spectra with flat top while the A and B QPOs are found where the power spectra exhibits weak power-law noise. In this paper, we study the C-type QPOs, as unlike A and B QPOs they increase in frequency during the rising phase of an outburst and decrease in frequency during the decay phase of the outburst.

As mentioned above C-type LFQPOs show a time evolution and can be considered to have origins based on shock propagation. The Propagating oscillatory shock (POS) model (Chakrabarti et al. 2005; Chakrabarti et al. 2008; Iyer et al. 2015) explains the time evolution of C-type QPOs as a function of shock location. The shock location is essentially the size of the post-shock corona region around the $\mathrm{BH}$. The model considers that QPOs are generated by the oscillation of this region. POS model is given by the equation,

$$
\begin{aligned}
v_{q p o} & =\frac{c}{2 \pi R r_{g} r_{s} \sqrt{r_{s}-1}} \\
\text { and } r_{s} & =r_{s o}-\frac{v t+\frac{a t^{2}}{2}}{r_{g}},
\end{aligned}
$$

where $v_{q p o}$ is the QPO frequency, $R$ is the shock com- 


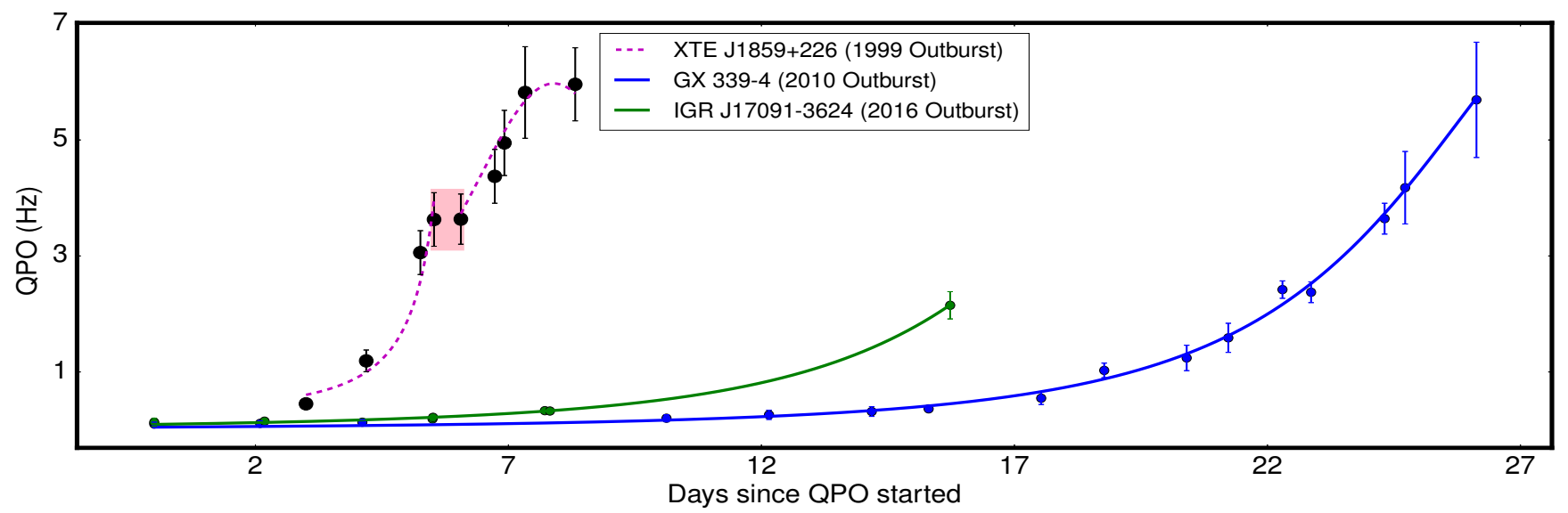

Figure 3. Evolution of QPO frequencies of XTE J1859+226 during its 1999 outburst, GX 339-4 during its 2010 outburst and IGR J17091-3624 during its 2016 outburst are shown here alongwith the fitted model. The shaded region (for XTE $\mathrm{J} 1859+226$ ) shows a QPO frequency which did not evolve for around half a day.

pression ratio or shock strength, $v$ is the velocity of the shock front, $a$ is the acceleration, $r_{s}$ is the instantaneous shock location and $r_{s o}$ is the initial shock location given in units of $r_{g}=\frac{2 G M}{c^{2}}$.

This model implicitly depends on mass of the compact object and hence can be used to estimate the black hole mass from the time evolution of QPOs. Typically the time evolution of QPOs are smooth without any discontinuity. In Figure 3, we show the case for XTE J1859+226 during its 1999 outburst. Here, we see that initially the QPO frequency increases with time, and then breaks and halts for a while. It then proceeds again with a smooth variation. The two frequencies in the shaded region of Figure 3 corresponding to the days MJD 51464.10 and MJD 51464.63 are identical as well as their power spectra are overlapping as is shown in Figure 4. From here we infer that the QPO parameters like its frequency, width, normalization and rms are also identical during this period. Examining the energy spectra corresponding to the two observations shaded in pink, we find that the spectral parameters are also identical in this period. This suggests that the accretion process has been steady and has not evolved from this state for a while. Besides this the QPO evolution for this outburst started off with an offset of 3 days as was required for the curve fitting using POS model. This indicates that the QPO evolution has started off earlier, but we do not have the data as the source was not observed in that period. The other two C-type LFQPO evolutions in the Figure 3 are corresponding to GX 339-4 (2010 outburst) and IGR J17091-3624 (2016 outburst). This shows that QPO evolution for different outbursts extends for different durations. The QPO evolution of XTE J1859+226 is steep while that for GX 339-4 is gradual. Also we notice that in the case of IGR J17091-
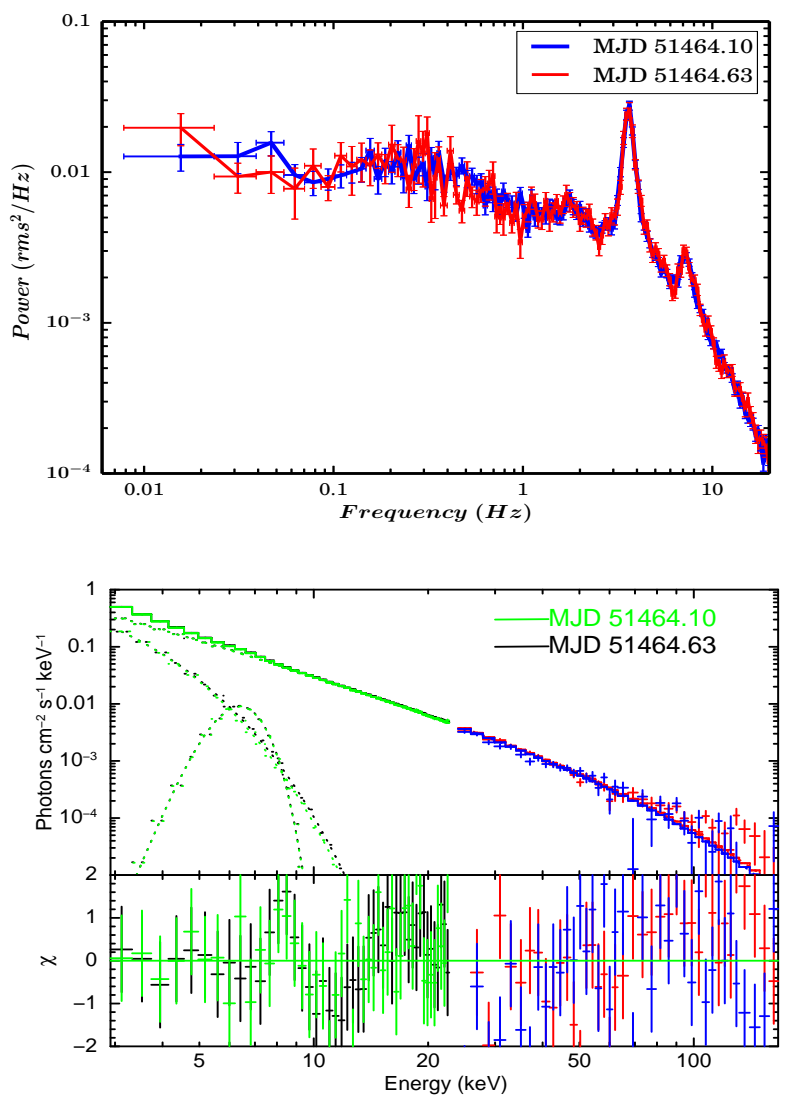

Figure 4. The power spectra (top) and the energy spectra (bottom) of the observations corresponding to the shaded region in Figure 3 are plotted. Here, the green and blue lines respectively indicates the PCA and HEXTE spectrum on MJD 51464.10 while the black and red lines corresponds to the observation on MJD 51464.63. It is evident that the system did not evolve during this period as both energy spectra and power spectra are almost identical. 
3624 the maximum frequency reached only up till 2.1 $\mathrm{Hz}$ while for the other two sources the maximum frequency reached around $6 \mathrm{~Hz}$.

The results of POS modelling for the three sources are presented in Table 1. For XTE J1859+226, modelling its QPO evolution gave a shock location of $206 r_{g}$, initial acceleration of $7.42 \times 10^{-06} \mathrm{~m} / \mathrm{s}^{2}$ followed by a deceleration of $1.60 \times 10^{-05} \mathrm{~m} / \mathrm{s}^{2}$ after the halt and a mass of $5.35 \pm 0.9 M_{\odot}$. Here the modelling is different from Radhika \& Nandi (2014) where they considered two POS fits for the two sections of QPO evolution before and after the halt and no acceleration was used. For the source GX 339-4, 2010 outburst the shock velocity is $10.73 \pm 2 \mathrm{~m} / \mathrm{s}$, constant deceleration is $1.45 \times$ $10^{-06} \mathrm{~m} / \mathrm{s}^{2}$, the initial shock location is $437 r_{g}$ and the estimated mass is $10.65 \pm 1.67 M_{\odot}$. For the source IGR J17091-3624, we obtained an initial shock location of $297 r_{g}$, initial velocity of $9.93 \pm 1.39 \mathrm{~m} / \mathrm{s}$, a constant deceleration of $1.81 \times 10^{-06} \mathrm{~m} / \mathrm{s}^{2}$ and mass of $11.08 \pm 0.79 M_{\odot}$. Below, we discuss about the evolution of spectral parameters of different states associated with outburst phases of the black hole binary sources.

\subsection{Evolution of Spectral States}

Outbursting sources transit through different states as was introduced earlier. The spectral evolution of the source XTE J1859+226 has been observed to be similar to typical GBH binaries. The spectra could be modelled using disk and powerlaw components. The source occupied all the spectral states of LHS, HIMS, SIMS and a short duration of HSS, and did complete the q-profile in its HID (See Figure 2). The temporal properties revealed the presence of type A, B, C and $\mathrm{C}^{*}$ (in decay phase) QPOs. Detailed study on this source have been discussed in Radhika \& Nandi (2014).

The energy spectra from the LHS and HSS of XTE $\mathrm{J} 1859+226$ has been shown in Figure 5. The PCA (RXTE) energy spectral data is plotted in black while the HEXTE (RXTE) spectral data is plotted in red. The dotted lines indicate the contribution from components used in the model like diskbb and powerlaw. For the LHS, we fitted the spectra with the model phabs(smedge*cutoffpl). The smeared edge was at $7.16 \mathrm{keV}$ and the high energy cut-off was at $50 \mathrm{keV}$. The photon index of the spectrum was $1.61 \pm 0.04$. For the HSS, we required a diskbb at $0.75 \mathrm{keV}$ and a powerlaw with index $2.39 \pm 0.18$. The HSS spectrum extended only up till $25 \mathrm{keV}$ and no cutoff was required.

We have modelled the energy spectrum of the source GX 339-4 as it went through the LHS, HIMS, SIMS and HSS in its 2002 outburst. It was observed that the spectra could be modelled with a powerlaw alone for the hard states while we required an additional disk component (diskbb) to model the hard intermediate and
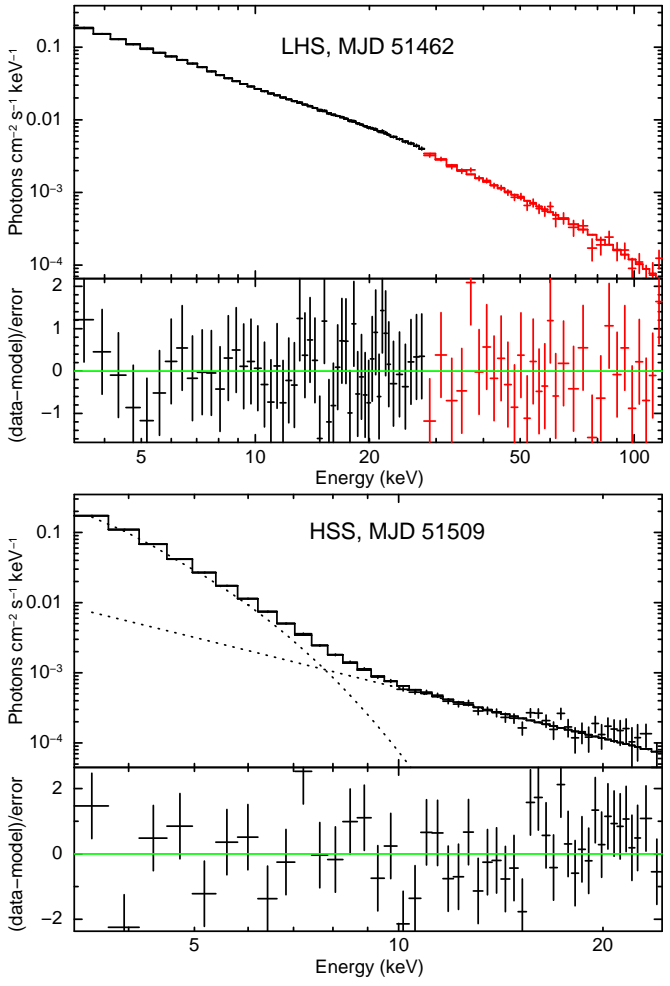

Figure 5. Phenomenological modelling of RXTE (PCAHEXTE) spectra in LHS and HSS of the source XTE J1859+226 during its 1999 outburst. No signature of hard $\mathrm{X}$-ray emission in HSS. The black lines are spectral data from RXTE-PCA and the red lines from RXTE-HEXTE instrument.
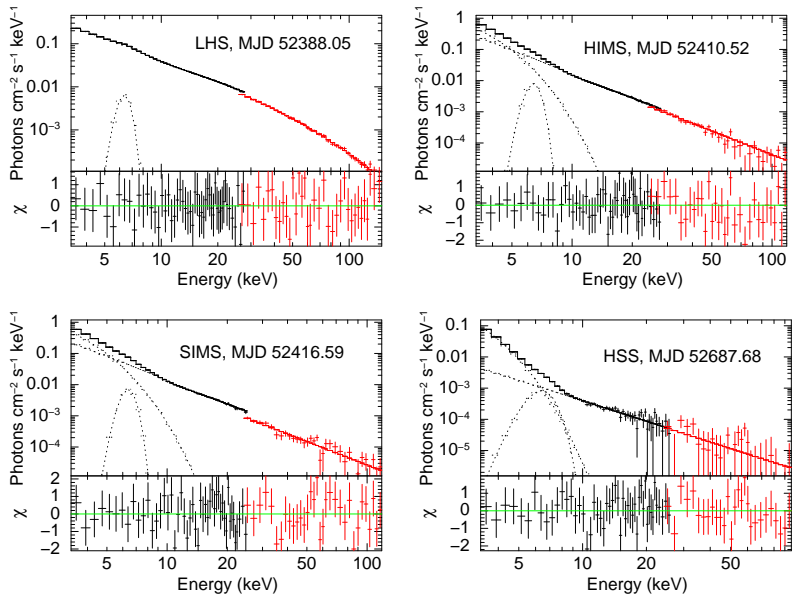

Figure 6. Phenomenological modelling of energy spectra corresponding to the four states of LHS, HIMS, SIMS and HSS of GX 339-4 during its 2002 outburst. The black curves are from RXTE-PCA $(3-25 \mathrm{keV})$ and the red curves are from RXTE-HEXTE (25-150 keV). The dotted lines indicate the contribution from model components like diskbb, gaussian and powerlaw. 
Table 1. Fit parameters from the POS model

\begin{tabular}{lccccr}
\hline Source & Outburst & Mass $\left(M_{\odot}\right)$ & $x_{s}\left(r_{g}\right)$ & Initial Velocity $(\mathrm{m} / \mathrm{s})$ & Acceleration $\left(\mathrm{m} / \mathrm{s}^{2}\right)$ \\
\hline XTE J1859+226 & 1999 & $5.35 \pm 0.9$ & 206 & $0 \pm 0.10$ & $7.42 \times 10^{-06},-1.60 \times 10^{-05}$ \\
GX 339-4 & 2010 & $10.65 \pm 1.67$ & 437 & $10.73 \pm 2$ & $-1.45 \times 10^{-06}$ \\
IGR J17091-3624 & 2016 & $11.08 \pm 0.79$ & 297 & $9.93 \pm 1.39$ & $-1.80 \times 10^{-06}$ \\
\hline
\end{tabular}
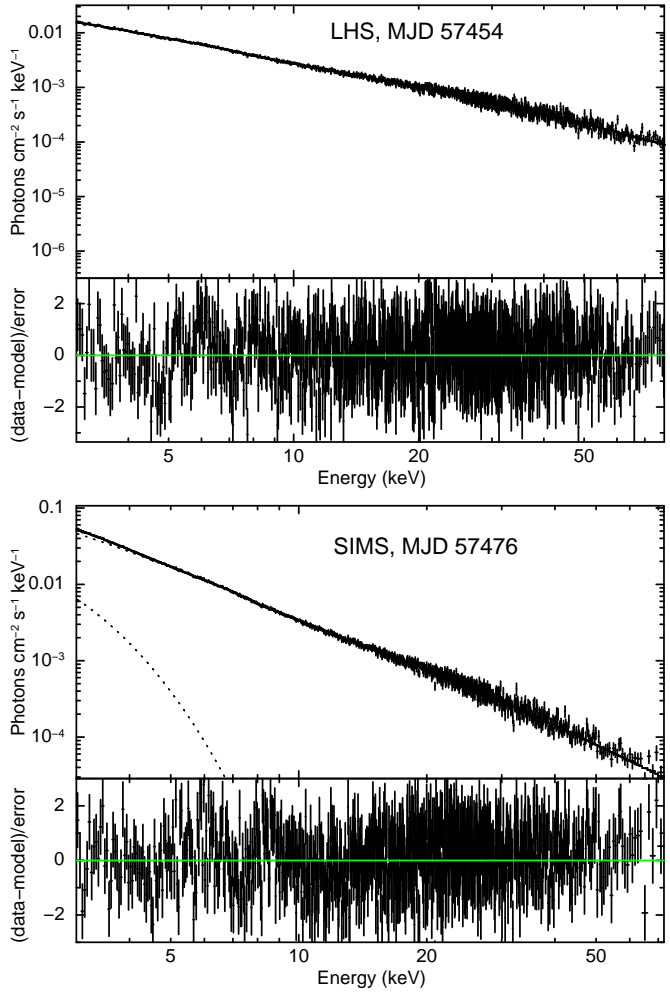

Figure 7. Phenomenological modelling of NuSTAR spectra in LHS and SIMS of the source IGR J17091-3624 during its 2016 outburst. The general model used for fitting the energy spectra is phabs(diskbb+ireflect $*$ cutoffpl). soft states. The power-law index, $\Gamma$ for the LHS was 1.58 which corresponds to a flat spectrum. For the HIMS and SIMS, the photon index was 2.51 and 2.52 respectively and for $\mathrm{HSS}, \Gamma \sim 2.18$. Though the value of $\Gamma$ is lower than expected for HSS, it should be noted that the percentage contribution of the total flux by diskbb in this state was $76.47 \%$. The presence of diskbb in the model indicates a significant contribution of the multitemperature blackbody emission with an inner disk temperature of $0.60 \mathrm{keV}$ and confirms that the source is in HSS. Figure 6 shows the phenomenological modelling of all four states of the source GX 339-4 during its 2002 outburst observed by $R X T E$.

IGR J17091-3624 during its 2016 outburst was found to occupy the LHS, HIMS and SIMS and only a very short presence of HSS (lasting for only a day)(Radhika et al. 2018). We have included two energy spectra from the 2016 outburst of this source corresponding to the LHS and SIMS in Figure 7. These are co-ordinated NuSTAR observations. The energy spectra of IGR J170913624 was modelled using disk, ireflect and cutoffpl components. We obtained a relative reflection value of $0.39 \pm$ 0.04 and $0.60 \pm 0.05$ for LHS and SIMS respectively. The photon index for LHS was $1.58 \pm 0.01$ and for the SIMS it was $2.41 \pm 0.02$. The modelling of simultaneous XRT-NuSTAR observations and details of the entire outburst (2016) including state classification, variability and mass estimation will appear in Radhika et al. (2018).

\subsection{Connection between QPO, Spectral States and Jet Ejection}

Radio jets are a very common phenomena in the black hole accretion process. During 'soft' X-ray states, the radio emission is strongly suppressed while jets are observed during the 'hard' states (Fender et al. 2004; Fender et al. 2009). Relativistic jets are observed as the system changes states from HIMS to SIMS. As a typical example, we have shown the case of the source XTE J1859+226 during its 1999 outburst in the Figure 2 with the location of one of its radio flares marked in red. A detailed study on the radio flares and connection with spectral states is presented in Radhika \& Nandi (2014).

We observed that QPOs are absent during the period of multiple jet ejections or flaring of the source 
XTE J1859+226 (Radhika \& Nandi 2014; Radhika et al. 2016a) have shown that type C LFQPOs appear around a day before the jet ejection. There is no presence of QPOs when the flare occurs, while type B QPOs are observed a few hours later. During the flares there is no phase lag observed between the soft and hard $\mathrm{pt}$ tons. When comparing the flux contributions, it $\mathrm{r}$ been observed that disk flux dominates over the nc thermal flux during the flares. In the LHS and HIN. the type C QPO frequency increases as both the $\mathrm{d}$ and powerlaw flux increases. In the rising phase as $\mathrm{w}$ as in the declining phase of the outburst strong rac jets have been observed. During the SIMS, it is fou that the type B QPO frequency is correlated with po erlaw flux, but not with disk flux. This indicates tl type B QPOs originate from the corona. It has also be noted that a few of the type C QPOs in SIMS are $\mathrm{r}$ correlated with the powerlaw flux while all $\mathrm{C}^{*} \mathrm{QP}$ are correlated only with the disk flux. Besides this 1 power density spectra had very low rms during the fla

Similarly in GX 339-4 during the 2002 and 20 outbursts, absence of QPOs have been found duri the time of a radio flare was detected (Radhika et 2016a). The correlation of radio flux with X-Ray fl for the source GX 339-4 has been studied in detail Corbel et al. 2013. Due to lack of radio observatic of IGR J17091-3624 during its outbursts, we could $\mathrm{r}$ explore this characteristic of disk-jet coupling.

\subsection{X-Ray Variability}

Next we look into the X-ray variability exhibited the three sources that we are studying. Here, we c ditionally studied the variabilities of GRS $1915+105$ a reference source as it is known for its 'class' variat ities. Figure 8 shows the variability exhibited by 1 sources GRS 1915+105 (in 1997), IGR J17091-36 (in 2016), XTE J1859+226 (in 1999) and GX 33s (in 2002, 2007 and 2010). Belloni et al. (2001) p. sented detailed analysis of the different classes of var1ability displayed by the source GRS $1915+105$. We have shown in Figure 8 the $\rho, \kappa$ and $\theta$ class variability of GRS $1915+105$ respectively. Thus, we see variability at different time scales $(\sim 50 \mathrm{~s}, \sim 100 \mathrm{~s}$ and $\sim 600 \mathrm{~s})$ for GRS $1915+105$. We compare it with the lightcurves of other sources and find that XTE J1859+226 does not have a well defined structure in its lightcurves while IGR J17091-3624 and GX 339-4 show some structure or repetitions in their lightcurves. The variabilities observed in GX 339-4 are not similar to any of the observed 'class' in GRS $1915+105$. It may be a combination of the $\rho$ and $\kappa$ classes exhibited by GRS $1915+105$. This requires further investigation.

We did not find any signature of variabilities during the LHS and HIMS. During the SIMS, we observed

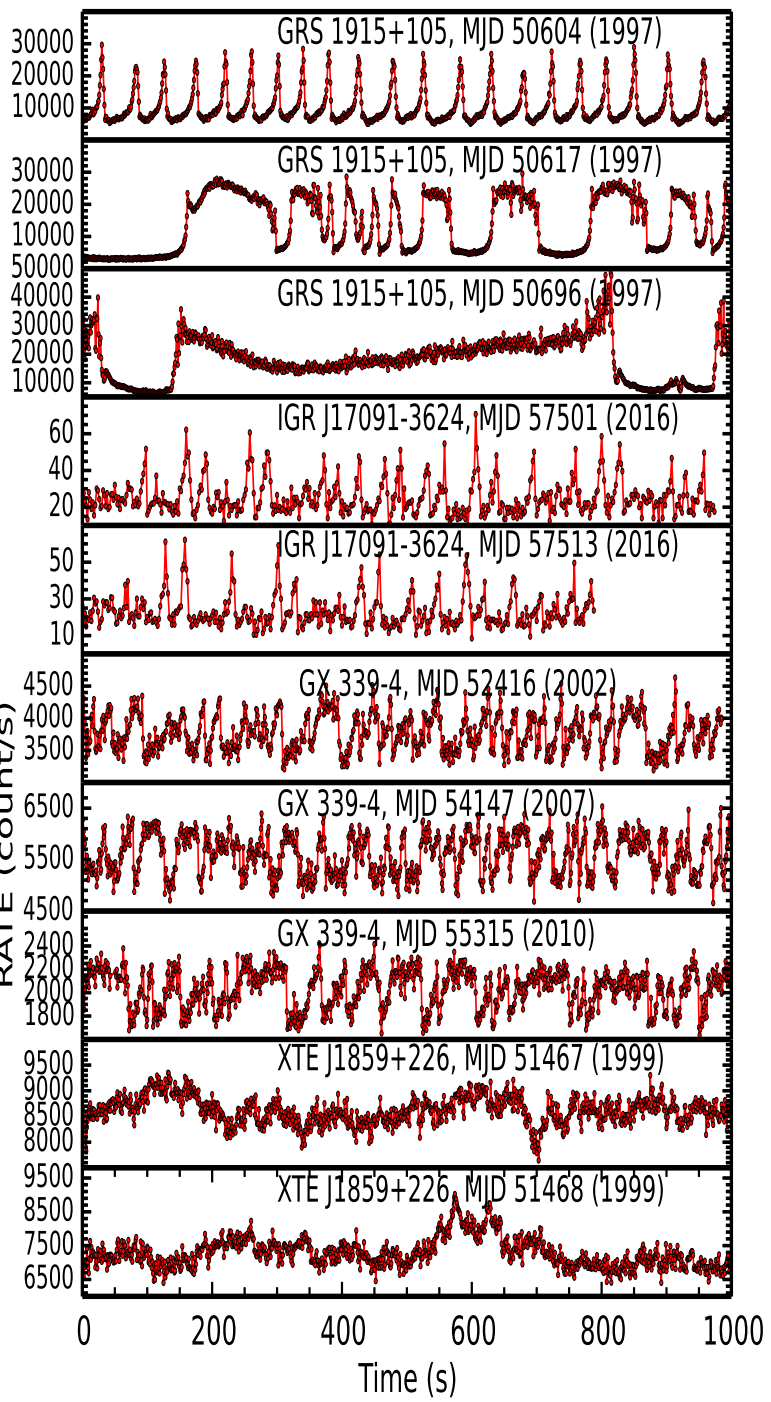

Figure 8. Structured variability exhibited by different $\mathrm{BH}$ sources during their outbursts. The first three panels show three of the variabilities exhibited by GRS $1915+105$ corresponding to $\rho, \kappa$ and $\theta$ classes respectively. The following panels compare it with the variabilities of IGR J17091-3624 (2016), GX 339-4 (2002, 2007 and 2010) and XTE J1859+226 (1999). The variabilities exhibited by the latter three sources were during the period of SIMS which gives us reasonable evidence that GRS $1915+105$ probably 'locked' in one of its intermediate states. 
that the IGR J17091-3624 exhibits variabilities in the X-ray lightcurve (Radhika et al. 2018) similar to that displayed by the same source in its 2011 outburst (Capitanio et al. 2012; Iyer et al. 2015). In HSS also the system showed oscillations, though weaker than that during the SIMS. It is noticed that IGR J17091-3624 is very faint as compared to the other sources that we study. Similarly in XTE J1859+226 and GX 339-4, we find signatures of variabilities with different time-scale when the source has transited to the SIMS.

It is also observed that for these sources the variability is observed only in lightcurves corresponding to the intermediate spectral states. Thus, we infer that GRS $1915+105$ possibly 'locked' in one of its intermediate states as it shows structured variability over the last two decades.

\section{Broadband Spectral Modelling with Two Com- ponent Flow - Mass Estimation}

The two component advective flow (Chakrabarti \& Titarchuk 1995; Chakrabarti \& Mandal 2006; Iyer et al. 2015) model incorporates a Keplerian disk (Shakura \& Sunyaev 1973) as well as a sub-keplerian halo around the black hole. The Keplerain disk is in the equatorial region and the sub-Keplerian halo is on top and bottom sides of the disk. During the hard states, the sub-Keplerian component predominates the flux contribution while during the soft states the major flux contribution is from the Keplerian disk. Here unlike in phenomenological models, the high and low energy photon emission are dependent on each other. As the outburst progresses, the effect of the sub-Keplerian component reduces and the disk starts contributing more to the total flux of the source.

We self-consistently calculate the total radiation spectrum from hydrodynamics (Mandal \& Chakrabarti 2005) and import the two component advective flow model as an additive table in XSPEC (Iyer et al. 2015). For each source under consideration there are four variable parameters in this model. They are the mass of the black hole $\left(M_{B H}\right)$ in units of $M_{\odot}$, shock location $\left(r_{s}\right)$ in units of $r_{g}$, keplerian disk accretion rate, $\dot{m}_{d}$ and subkeplerian halo accretion rate $\left(\dot{m}_{h}\right)$ in units of Eddington rate. Besides this we have the norm parameter which is a constant for a source as it is dependent on distance to the source, its mass and inclination. For the fitting, we use spectra from 3 to $150 \mathrm{keV}$. During the softer states as the higher energy range of the spectral data decreases we fit only up to $80 \mathrm{keV}$ or so. We perform fitting with the two component model for all data sets of an outburst of the source leaving the 'norm' parameter free. Then we take an average of all the norms and fix it as the norm for the source. Fit results for both phenomenological and two component models for all three sources under consideration are presented in Table 2 .
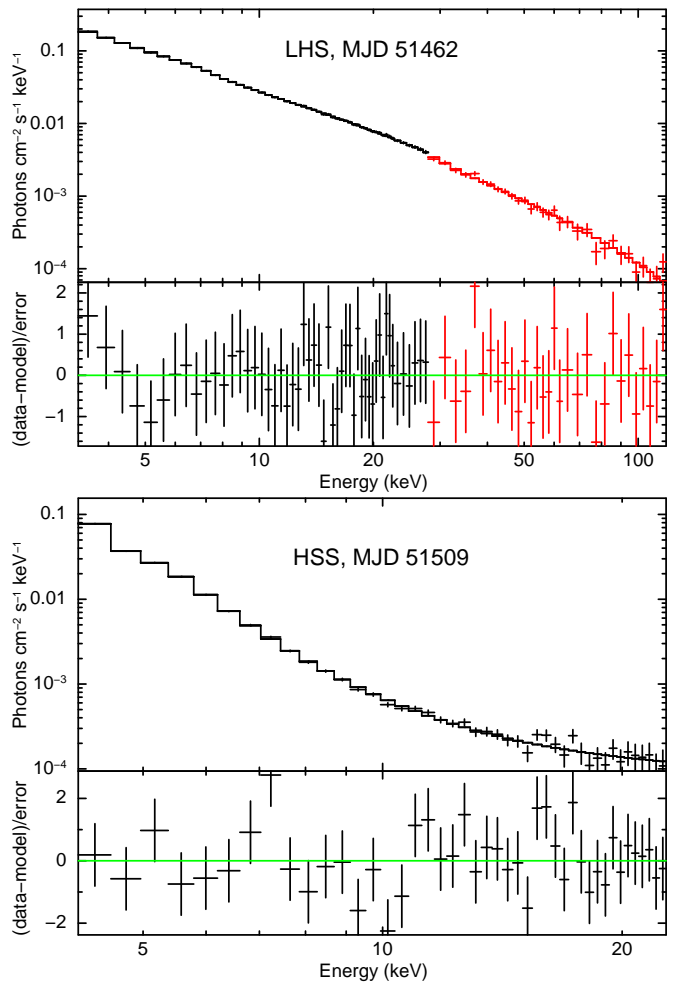

Figure 9. Two component flow modelling of RXTE (PCA-HEXTE) spectra in LHS and HSS of the source XTE J1859+226 during its 1999 outburst.

Figure 9 shows the energy spectra of the source XTE J1859+226 during its 1999 outburst modelled with the two component flow. We present results only from two states (LHS and HSS) in this paper. Detailed analysis of each state of the outburst is presented in Nandi et al.(2018). When the source was in the LHS (on MJD 51462), it had a halo accretion rate of $0.26 \dot{M}_{E d d}$ and a Keplerian accretion rate of $0.15 \dot{M}_{E d d}$. The shock location was at $101 \pm 2 r_{g}$ and the mass of the source is estimated as $6.0 \pm 0.53 M_{\odot}$. In the HSS, the accretion rates are $0.18 \dot{M}_{E d d}$ for the halo and $0.49 \dot{M}_{E d d}$ for the disk. The computation of shock location gave a value of $24.6 \pm 1.2 r_{g}$. The mass estimate for the source from this model is $6.05 \pm 0.27 M_{\odot}$.

In Figure 10, we have shown the two component flow based spectral modelling performed for GX 3394 during its 2002 outburst. Here, we find that during the LHS the shock location is $424 \pm 7 r_{g}$, halo rate is $0.20 \dot{M}_{E d d}$ and disk rate is $0.04 \dot{M}_{E d d}$. As the source moves to HIMS, the shock location becomes $190 \pm 12$ $r_{g}$. The halo rate declines to $0.06 \dot{M}_{E d d}$ and while disk rate increases to $3.3 \dot{M}_{E d d}$. In SIMS, the shock location 
Table 2. Spectral fit parameters using phenomenological and two component flow models

\begin{tabular}{|c|c|c|c|c|c|c|c|c|c|}
\hline Source & MJD (State) & Observatory & Tin $(\mathrm{keV})$ & rel_refl & Photon Index & high-cut $(\mathrm{keV})$ & $\dot{m}_{h}, m_{d}\left(\dot{M}_{E d d}\right)$ & Mass $\left(M_{\odot}\right)$ & $x_{s}\left(r_{g}\right)$ \\
\hline & & & \multicolumn{3}{|c|}{ Phenomenological } & & \multicolumn{3}{|c|}{ Two component flow } \\
\hline XTE J1859+226 & 51462 (LHS) & PCA-HEXTE & & & $1.61 \pm 0.04$ & $50 \pm 8.0$ & $0.26,0.15$ & $6.0 \pm 0.053$ & $101 \pm 2$ \\
\hline XTE J1859+226 & 51509 (HSS) & PCA & $0.75 \pm 0.005$ & - & $2.39 \pm 0.18$ & - & $0.18,0.49$ & $6.05 \pm 0.27$ & $24.6 \pm 1.2$ \\
\hline GX 339-4 & 52388 (LHS) & PCA-HEXTE & - & $0.39 \pm 0.17$ & $1.58 \pm 0.04$ & $71 \pm 6$ & $0.20,0.04$ & $11.56 \pm 0.17$ & $424 \pm 7$ \\
\hline GX 339-4 & 52410 (HIMS) & PCA-HEXTE & $0.86 \pm 0.01$ & - & $2.52 \pm 0.02$ & - & $0.06,3.3$ & $11.02 \pm 0.14$ & $190 \pm 12$ \\
\hline GX 339-4 & 52416 (SIMS) & PCA-HEXTE & $0.88 \pm 0.03$ & - & $2.51 \pm 0.07$ & - & $0.04,0.57$ & $10.21 \pm 0.35$ & $46 \pm 2$ \\
\hline GX 339-4 & 52687 (HSS) & PCA-HEXTE & $0.60 \pm 0.05$ & - & $2.18 \pm 0.11$ & - & $0.64,10.6$ & $9.55 \pm 0.47$ & $5 \pm 0.03$ \\
\hline IGR J17091-3624 & 57454 (LHS) & NuSTAR & - & $0.39^{+0.06}$ & $1.58^{+0.028}$ & $228_{-44}^{+72}$ & $0.36,0.04$ & $11.66 \pm 0.12$ & $484 \pm 18$ \\
\hline IGR J17091-3624 & 57476 (SIMS) & NuSTAR & $0.59_{-0.015}^{+0.014}$ & $0.60_{-0.08}^{+0.89}$ & $2.41_{-0.020}^{+0.017}$ & - & $0.07,0.19$ & $11.51 \pm 0.07$ & $56 \pm 2$ \\
\hline
\end{tabular}
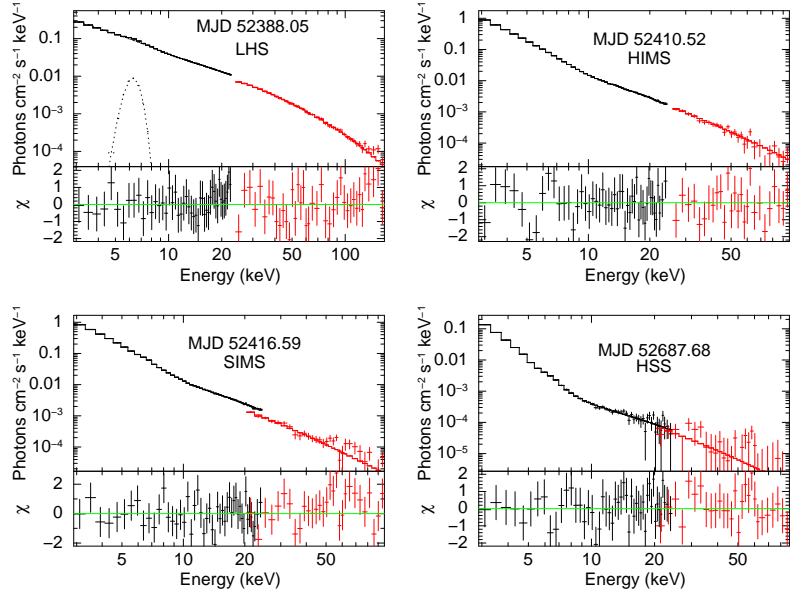

Figure 10. Two component flow modelling of energy spectra corresponding to the four states of GX 339-4 during its 2002 outburst. The black lines correspond to spectral data from PCA, the red lines correspond to spectral data from HEXTE and the dotted lines are the model components like gaussian and two component flow.

has reduced further to $46 \pm 2 r_{g}$, the halo rate is 0.04 $\dot{M}_{E d d}$ and the disk rate increased to $0.57 \dot{M}_{E d d}$. Finally, in the HSS we obtained the limiting value of shock location of $5 r_{g}$, a halo rate of 0.64 and a disk rate of 10.6 both in units of $\dot{M}_{E d d}$. We also estimated the mass from spectral modeling with two component flow and found that GX 339-4 mass lies in the range 9.20 to $11.95 M_{\odot}$. Heida et al. (2017) has estimated the black hole mass of GX 339-4 to be between $2.3 M_{\odot}$ and $9.5 M_{\odot}$. Detailed broadband spectral modelling of all outbursts of GX 339-4 is under progress and the results will be presented elsewhere.

Similarly, we have studied the source IGR J170913624 during its 2016 outburst by means of two component flow modelling of SWIFT and NuSTAR simultaneous spectra (Radhika et al. 2018). During the LHS, the shock location obtained was $484 r_{g}$ and it reduced to $56 r_{g}$ as the source entered the SIMS. The halo accretion rate changed from 0.36 to $0.07 \dot{M}_{E d d}$ and the disk accretion rate increased from 0.04 to $0.19 \dot{M}_{E d d}$. The mass estimates from the two observations are $11.66 \pm$ 0.12 and $11.51 \pm 0.07 M_{\odot}$.
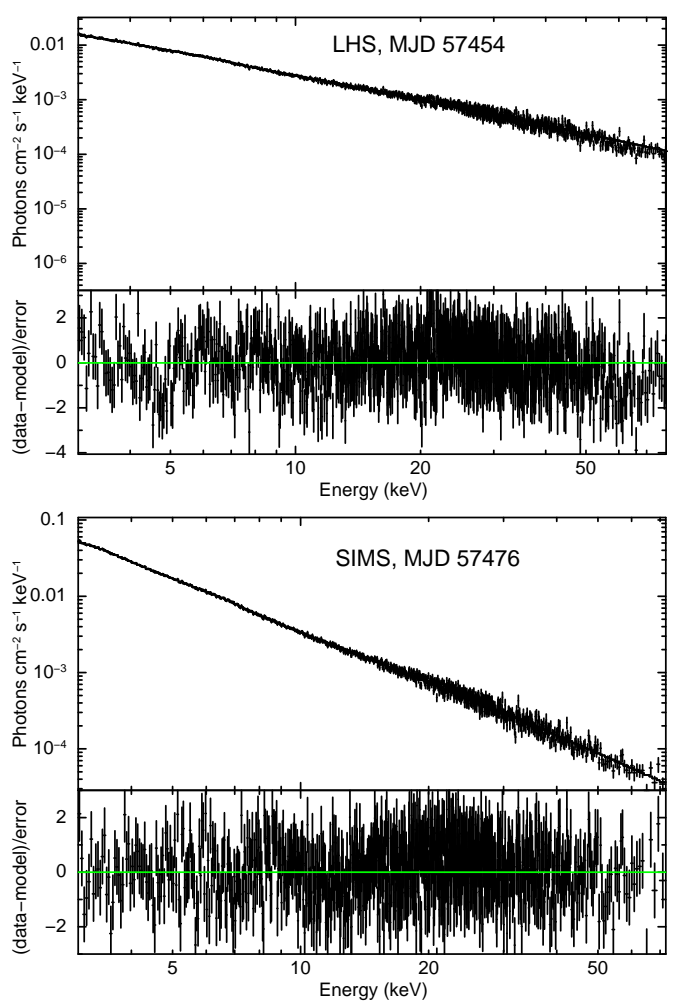

Figure 11. Two component flow modelling of NuSTAR spectra in LHS and SIMS of the source IGR J17091-3624 during its 2016 outburst. 
Figure 11] shows the energy spectra of IGR J170913624 during the LHS and SIMS of its 2016 outburst as observed by NuSTAR. Thus we could understand the spectral evolution of the different sources based on two component advective flow modelling and estimate the mass of the compact objects in these systems.

\section{Conclusion and Future Work}

In this paper, we present the results of evolution of spectro-temporal characteristics of three different GBH binaries. First, we present the frequency of outbursts for different sources that are quite different from each other. Some sources like GX 339-4 are dynamic whereas sources like XTE J1859+226 remains in a quiescent phase for most of the time. The evolution of outburst profile of these sources show that they do not have a unique variation of the intensity. The profiles are found to be either a FRED or slow rise and exponential decay.

We have studied how the black hole binary sources evolve along the hardness intensity diagram (q-plot) during their outburst. The sources XTE J1859+226 and GX 339-4 are observed to complete the q-profile and hence exhibit all the spectral states. IGR J17091-3624 is found to be different from this typical variation and do not occupy all the spectral states. The 2016 outburst of IGR J17091-3624 has just one observation corresponding to a possible HSS wherein the hardness ratio was a minimum and the signature of disk was prominent (Radhika et al. 2018).

We have also modelled the energy spectra using the two component flow model and found the variation of spectral characteristics to be matching with that obtained using phenomenological modelling. With the help of this, we have been able to find that the shock location decreases as a source evolves from its LHS to HSS. Additionally, the variation of halo rate and disk rate agrees well with the variation of soft and hard fluxes respectively. During the LHS, the halo rate is found to be maximum and decreases as the source approaches HSS. Similarly, the disk rate increases when the source energy spectra softens towards the HSS (Radhika et al. 2018).

The temporal characteristics show the presence of LFQPOs in the power spectra. Different types like A, $\mathrm{B}, \mathrm{C}$ and $\mathrm{C}^{*}$ have been identified for the different sources. The $\mathrm{C}$ type QPO frequency is observed to increase as the outburst progresses. We also modelled the time evolution with the help of the POS model and understand that the QPO is generated by the propagation of a shock front (see Figure 3).

There also exists a possible connection between the occurrence of radio flares and spectro-temporal char- acteristics of the sources. Figure 2 shows the location of radio flare for the 1999 outburst of the source XTE $\mathrm{J} 1859+226$. We find that during the period the flare occurs as indicated by radio lightcurves, the power spectra does not show presence of QPOs and the total rms variability decreases (Radhika \& Nandi 2014). The energy spectra is observed to get softer as evident in increase of soft flux (disk rate) and decline in the hardness ratio. This suggest that probably the innermost part of the disk (hot corona) is being evacuated into jets/flares, resulting in the soft flux to dominate. Hence, the absence of any oscillations of the corona leads to no detection of QPOs. Thus, we can be certain that the origin of QPOs is linked with the oscillations of the corona.

An interesting feature observed in all the sources studied in this paper, is the occurrence of variability signatures in the X-ray lightcurves. These are found to be similar to that observed in GRS 1915+105 (Belloni et al. 2001) and IGR J17091-3624 (2011 outburst; Altamirano et al. 2011). We find that the variability signatures are not identical for all the sources studied here. Additionally, a comparative study with the spectral evolution suggest that these variabilities appear at the time the source transits to the intermediate state (specifically SIMS in XTE J1859+226 and GX 339-4). We consider this as an indication that GRS $1915+105$ which exhibits different 'class' variabilities is probably 'locked' in one of the intermediate states.

Finally, we also modelled the energy spectra of the three sources using the two component advective flow paradigm and estimated the Keplerian and sub-Keplerian accretion rates, shock location and mass of the compact objects. These results are presented in Table 2.

In this paper, we have not considered the effects of black hole spin in order to understand the spectrotemporal evolution. As a future study we intend to do the analysis of QPOs and jet ejection mechanisms in the Kerr space. Broadband analysis and modelling of the energy spectra considering the rotational effects of the compact object can shed new light on the origin of QPOs. Moreover, the effect of black hole spin on the radio flaring of the BHBs will also be studied in detail.

\section{Acknowledgements}

We are thankful to the reviewer whose valuable suggestions have helped in improving the manuscript. AN thanks GD, SAG; DD, PDMSA and Director, ISAC for encouragement and continuous support to carry out this research. This research has made use of the data obtained through High Energy Astrophysics Science Archive Research Center online service, provided by NASA/Goddard Space Flight Center. 


\section{References}

[1] Altamirano, D., T. Belloni, M. Linares, M. van der Klis, R. Wijnands, P. A. Curran, M. Kalamkar et al. 2011, The Astrophysical Journal Letters, 742.2 , L17

[2] Altamirano, D., Linares, M., van der Klis, M., et al. 2011b, ATel, 3225

[3] Belloni T., M endez M., S anchez-Fern andez C., 2001, $A \mathcal{E} A, \mathbf{3 7 2}, 551$

[4] Belloni, T and Homan, J and Casella, P and Van Der Klis, $\mathrm{M}$ and Nespoli, El and Lewin, WHG and Miller, JM and Méndez, M, 2005, $A$ E A, 440-1, 207-222

[5] Capitanio, F., Bazzano, A., Ubertini, P., Zdziarski, A. A., Bird, A. J., De Cesare, G., Dean, A. J., Stephen, J. B., Tarana, A., 2006, ApJ, 643, 376-380.

[6] Capitanio, F., Del Santo, M., Bozzo, E., Ferrigno, C., De Cesare, G., Paizis, A., 2012, MNRAS, 422, 31303141.

[7] Casella, Piergiorgio, Tomaso Belloni, Jeroen Homan, and Luigi Stella. 2004, $A \mathcal{E} A$, 426-2, 587-600.

[8] Corbel, S., Coriat, M., Brocksopp, C., Tzioumis, A. K., Fender, R. P., Tomsick, J. A., Buxton, M. M., Bailyn, C. D., 2013, MNRAS, 428, 2500-2515.

[9] Corral-Santana, Jesus M., Jorge Casares, Teo MunozDarias, Franz E. Bauer, Ignacio G. Martinez-Pais, and David M. Russell 2016 A $\mathcal{E}$ A, 587, A61.

[10] Chakrabarti, S. K., \& Mandal, S., 2006, The Astrophysical Journal Letters, 642, L49

[11] Chakrabarti, S.K., Titarchuk, L. G. 1995, ApJ, 455, 623

[12] Chakrabarti, Sandip K and Nandi, A and Debnath, D and Sarkar, R and Datta, BG. 2005, Indian J. Phys, 79. 8, 841-845.

[13] Chakrabarti, Sandip K., Dipak Debnath, Anuj Nandi, and P. S. Pal. 2008, $A \mathcal{E}$ A, 489-3, L41-L44.

[14] Fender, R.P., Belloni, T.M. and Gallo, E., 2004, $M N$ RAS, 355(4), 1105-1118.

[15] Fender, R. P., Homan, J., Belloni, T. M., 2009, MNRAS, 396, 1370-1382.

[16] Feng, H. and Soria, R. 2011, New Astronomy Reviews, 55.5, 166-183.

[17] Heida, M., Jonker, P. G., Torres, M. A. P., Chiavassa, A., 2017, ApJ, 846:132, 8pp

[18] Homan, Jeroen, Wijnands, Rudy, Van Der Klis, Michiel, Belloni, Tomaso, van Paradijs, Jan, Klein-Wolt, Marc, Fender, Rob, Mendez, Mariano. 2001, Astrophys. J. Suppl. Ser, 132-2, 377

[19] Homan J., Belloni T. 2005, Ap E SS, 300, 107

[20] Iyer, N and Nandi, A and Mandal, S. 2015, ApJ, 807-1, 108

[21] Motta, S., Muñoz-Darias, T., Casella, P., Belloni, T. and Homan, J. 2011, MNRAS, 418, 2292-2307

[22] Mandal, S. and Chakrabarti, S. K., 2005, A $\mathcal{F} A$, 434, 839

[23] Nandi, Anuj and Debnath, Dipak and Mandal, Samir and Chakrabarti, Sandip K. 2012, A $\mathcal{G}$ A, 542, A56
[24] Nandi, A., Mandal, S., Sreehari, H., Radhika, D., Das, S., Chattopadhyay, I., Iyer, N., Agrawal, V., Aktar, R. 2017, Ap $\mathcal{E} S S$ (under review)

[25] Podsiadlowski, Ph, Saul Rappaport, and E. D. Pfahl. 2002, ApJ, 565, no. 2, 1107.

[26] Radhika, D and Nandi, A. 2014, Advances in Space Research, 54-8, 1678-1697

[27] Radhika, D., Nandi, A., Agrawal, V. K., \& Seetha, S. 2016,MNRAS, 460, 4403

[28] Radhika, D., Nandi, A., Agrawal, V. K., \& Mandal, S. 2016, MNRAS, 462, 1834

[29] Radhika, D., Sreehari, H., Nandi, A., Iyer, N. and Mandal, S. 2017, MNRAS (under review)

[30] Remillard R. A., McClintock J. E. 2006, ARAEAA, 44, 49

[31] Remillard, Ronald A., Edward H. Morgan, Jeffrey E. McClintock, Charles D. Bailyn, and Jerome A. Orosz. 1999 The Astrophysical Journal, 522, no. 1, 397.

[32] Shakura, N. I., and Rashid Alievich Sunyaev. 1973 A E A, 24, 337-355.

[33] Strohmayer, Tod E. 2001The Astrophysical Journal Letters, 552, no. 1, L49.

[34] Tanaka, Y., \& Lewin, W. 1995, in X-Ray Binaries, Eds. W. Lewin, J. van Paradijs, \& P. van den Heuvel, Cambridge Univ. Press, Cambridge, 126

[35] Tanaka, Yasuo, and Noriaki Shibazaki. 1996 Annual Review of Astronomy and Astrophysics,34, no. 1, 607644.

[36] Tetarenko, B. E., G. R. Sivakoff, C. O. Heinke, and J. C. Gladstone. 2016, Astrophys. J. Suppl. Ser., 222, no. 2, 15 .

[37] Wood A., Smith D. A., Marshall F. E., Swank J. H. 1999, IAUC, $\mathbf{7 2 7 4}$ 